\title{
Health Information Exchange and its Barriers and Facilitators in Low- and Middle-Income Countries: Key Healthcare Stakeholders' Perceptions from Pakistan
}

\author{
Ather Akhlaq, Brian McKinstry, Aziz Sheikh \\ Centre for Medical Informatics \\ University of Edinburgh \\ FirstName.LastName@ed.ac.uk
}

\begin{abstract}
Reliable and timely health information is useful in making important health decision by providers and policy makers to enhance the healthcare quality of individual patients and the whole population. We describe an ongoing qualitative work to explore the deployment strategies for Health Information Exchange (HIE) in Pakistan. The preliminary findings from the analysis of the first 15 interviews presented an overview of evolved categories such as difficulties of using paper-based information systems, barriers and facilitators to HIE, advantages and disadvantages of HIE and the existence of fragmented HIE in Pakistan. Stakeholders found HIE a useful intervention to manage and analyse health data for better decision making and improved health outcomes.
\end{abstract}

Health information exchange, Low and middle income, Implementation, Adoption, Barriers, Facilitators

\section{INTRODUCTION}

Reliable and timely health information is necessary for effective health management and public health outcomes. Policy makers, healthcare professionals, industry groups and researchers recognise health information exchange (HIE) as a vital component of the solution to disparate, fragmented and noninteroperable health systems [1-2].

According to the WHO report on health information systems, countries that face the greatest health challenges generally have the weakest system for gathering, managing, analysing and using health information [3]. Unfortunately, the health systems responsible to accommodate health related challenges in low and middle-income countries (LMICs) are confronted with scarce resources and capability.

This study focuses on Pakistan, a LMIC, exploring perceptions of key healthcare stakeholders on the deployment and diffusion of HIE processes at community, district, provincial and/or national level.

\section{METHODS}

\subsection{Design}

We conducted semi-structured interviews with key healthcare stakeholders including citizens to determine their perceptions on the implementation and adoption of HIE.

\subsection{Sampling / Sampling Framework}

We used a purposive sampling technique to maximise the variation within the sample to ensure the views of as many different types of people were included [4]. Respondents were recruited based on their experiences and positions in the healthcare sector of Pakistan.

The sampling framework was reviewed constantly throughout the data collection process and was adapted, where necessary, to ensure the breadth and depth of coverage (see Table 1). Recruiting and interviewing continued until data saturation was attained.

\begin{tabular}{|l|}
\hline $\begin{array}{l}\text { Sampling Framework consists of the following } \\
\text { domains: }\end{array}$ \\
\hline Secondary Care \\
\hline Tertiary Care \\
\hline Primary Care \\
\hline Citizens \\
\hline eHealth / HMIS / Telehealth \\
\hline Government Officials /Private Officials \\
\hline Facility Directors / Administrators / Managers \\
\hline Rural Areas \\
\hline International Organisations \\
\hline $\begin{array}{l}\text { Charity Organisations /Non-Governmental } \\
\text { Organisation }\end{array}$ \\
\hline Health Professional \\
\hline
\end{tabular}

Table1. Sampling framework 


\section{Extended Abstracts.}




\subsection{Data Analysis}

We selected Thematic Analysis (TA) because it provides a description and understanding of answers and allows the discovering of patterns and themes. In TA, themes can be identified inductively and deductively and is a flexible method [5].

\section{PRELIMINARY RESULTS}

39 interviews from 43 participants have been conducted ( 2 were group interviews by chance), 32 have been transcribed, 15 have been coded.

\section{Evolved Categories:}

- Advantages of HIE

- disadvantages of HIE

- verbal, paper referrals

- HIE barriers

- HIE facilitators

- Electronic statistical health data transfer in public sector

- HIE within big organisations HIE across organisations

- International donor vertical programs

- Problems with manual data

- Data and information collection methods

- Verbal communication (patient to doctor, among doctors, among providers)

- Support from NGOs in public facilities

- mHealth may be useful

\section{DISCUSSION}

The findings showed a number of facilitators needed to overcome perceived barriers to implement and adopt HIE in Pakistan. Moreover, HIE was perceived to have more advantages than disadvantages and many benefits, such as low-cost integrated healthcare, could be realised through HIE in LMICs. mHealth would be a useful technology to implement HIE due to weak infrastructure and lack of technology in LMICs.

\subsection{Strengths and Limitations}

\section{Strengths:}

- Maximum variation sampling strategy

- Iterative approach to data analysis which involved both inductive and deductive analysis

- Interviewing until saturation was achieved
- Response rate $63 \% \quad$ (50 participants responded out of 79)

\section{Limitations:}

- Interviews were often conducted through Skype and Mobile/Telephone as it was not possible to travel to other parts of Pakistan for face to face conversation

- Difficult to get contacts of female participants from other participants (only 9 female interviews) because of cultural restrictions.

- Some of the interviews were taken in noisy public places (hotel, restaurant) due to inaccessible premises.

- High profile and busy participants who were unwilling to spend up to one hour in an interview.

\section{CONCLUSION}

In Pakistan, HIE existed within large organisations and the concept of inter-organisational HIE and interoperability was still in infancy. To overcome the barriers, there are many facilitators that can help enable HIE implementation and adoption. Stakeholders were positive about the technology and wanted to avail the advantages of HIE in the near future.

\section{Acknowledgements}

I thank the research participants for giving their time and valuable data for this research. I am thankful to Higher Education Commission (HEC) Pakistan and the University of Edinburgh for funding this $P h D$ project.

\section{REFERENCES}

[1] Brailer, D.J. Interoperability: the key to the future health care system. Health Aff (Millwood). 2005 Jan-Jun;Suppl Web Exclusives:W5-19-21.

[2] Hripcsak, G., Kaushal, R. et al. The United Hospital Fund meeting on evaluating health information exchange. Journal of biomedical informatics, Vol. 40 No. 6, pp. S3-S10, 2007.

[3] W orld Health Organization, 2011. Country health information systems: a review of the current situation and trends.

[4] Tongco, M. Purposive sampling as a tool for informant selection. Ethnobotany Research and Applications. Vol 5, 2007, p147-158

[5] Braun, V. \& Clarke, V. Using thematic analysis in psychology. Qualitative research in psychology, Vol. 3 No. 2, pp. 77-101, (2006). 